\title{
Diagnostic Evaluation in Patients with Intractable Epilepsy and Normal Findings on MRI: A Decision Analysis and Cost-Effectiveness Study
}

E. Widjaja, B. Li, and L. Santiago Medina

\begin{abstract}
BACKGROUND AND PURPOSE: Patients with focal intractable epilepsy and normal MR imaging findings frequently undergo further diagnostic tests to localize the epileptogenic zone. The aim of this study was to determine the cost-effective diagnostic strategy that will identify the epileptogenic zone in patients with suspected focal intractable epilepsy and normal MR imaging findings by using decision analysis.
\end{abstract}

MATERIALS AND METHODS: A Markov decision model was constructed by using sensitivities and specificities of test strategies, seizure outcomes following surgical and medical treatment, cost, utilities, probabilities, and standardized mortality ratios. We compared 6 diagnostic test strategies: PET, ictal SPECT, and MEG individually; and combinations of PET+SPECT, PET+MEG, and SPECT+MEG. The outcomes measured were health care costs, QALY, and ICER. One-way and probabilistic sensitivity analyses were conducted to adjust for uncertainties in model parameters.

RESULTS: The preferred strategies were PET+MEG and SPECT. The health care cost of the baseline strategy (PET+MEG) was $\$ 95,612$ with 16.30 QALY gained. SPECT cost $\$ 97,479$ with 16.45 QALY gained and an ICER of \$12,934/QALY gained compared with those in PET+MEG. One-way sensitivity analyses showed that the decisions of the model were sensitive to variations in sensitivity and specificity of the test strategies. Probabilistic sensitivity analysis showed that when the willingness to pay was $<\$ 10,000, P E T+M E G$ was the favored strategy, but the favored strategy changed to SPECT when the willingness to pay was $>\$ 10,000$.

CONCLUSIONS: PET+MEG and SPECT were the preferred strategies in the base case. The choice of test was dependent on the sensitivity and specificity of test strategies and willingness to pay. Further study with a larger sample size is needed to obtain better estimates of sensitivity and specificity of diagnostic tests.

ABBREVIATIONS: EEG = electroencephalography; ICER = incremental cost-effectiveness ratio; $M E G$ = magnetoencephalography; QALY = quality-adjusted life years; $S M R=$ standardized mortality ratio

A pproximately $20 \%-40 \%$ of patients with epilepsy become refractory to medications. ${ }^{1-3}$ Intractable epilepsy is associated with impaired quality of life ${ }^{4,5}$ and higher mortality rates. ${ }^{6-8}$ Epilepsy surgery offers the potential to render some of these patients seizurefree. ${ }^{9-12}$ The success of epilepsy surgery relies heavily on accurate identification of the epileptogenic zone. In patients with intractable epilepsy, extensive investigations are performed to identify suitable

Received July 5, 2012; accepted after revision July 24.

From the Diagnostic Imaging and Division of Neurology (E.W.), Hospital for Sick Children, Toronto, Ontario, Canada; Department of Health Policy Management and Evaluation (B.L.), University of Toronto, Toronto, Ontario, Canada; and Department of Radiology and Health Outcomes Policy and Economic Center (L.S.M.), Miami Children's Hospital, Miami, Florida.

This work was supported by the GE-AUR award.

Please address correspondence to Elysa Widjaja, MD, Diagnostic Imaging, Hospital for Sick Children, 555 University Ave, Toronto, Ontario M5G 1X8, Canada; e-mail: Elysa.Widjaja@sickkids.ca

= Indicates article with supplemental on-line tables

http://dx.doi.org/10.3174/ajnr.A3474 candidates who could benefit from epilepsy surgery, including scalp EEG and MR imaging. Patients with normal MR imaging findings have poorer surgical outcomes compared with those with a lesion identified on MR imaging. ${ }^{13-15}$ Those with suspected focal epilepsy on scalp EEG or clinical semiology and normal MR imaging findings would undergo further investigation to clarify the location of the epileptogenic zone. If further investigation clarifies the location of epileptogenic zone, these patients would undergo epilepsy surgery. Otherwise they would continue with medical treatment.

Three types of diagnostic tests are established in clinical practice to identify the epileptogenic zone: FDG-PET, ictal SPECT, and MEG. Although the sensitivity and/or specificity of the tests have been reported, ${ }^{16-20}$ the cost-effectiveness of these tests has never been examined. Given the high cost of these investigations and the current environment of restricted resources, their cost-effectiveness needs to be evaluated to assist in the decision-making process. The objective of this study was to determine the cost-effective diagnostic strategy that 
A

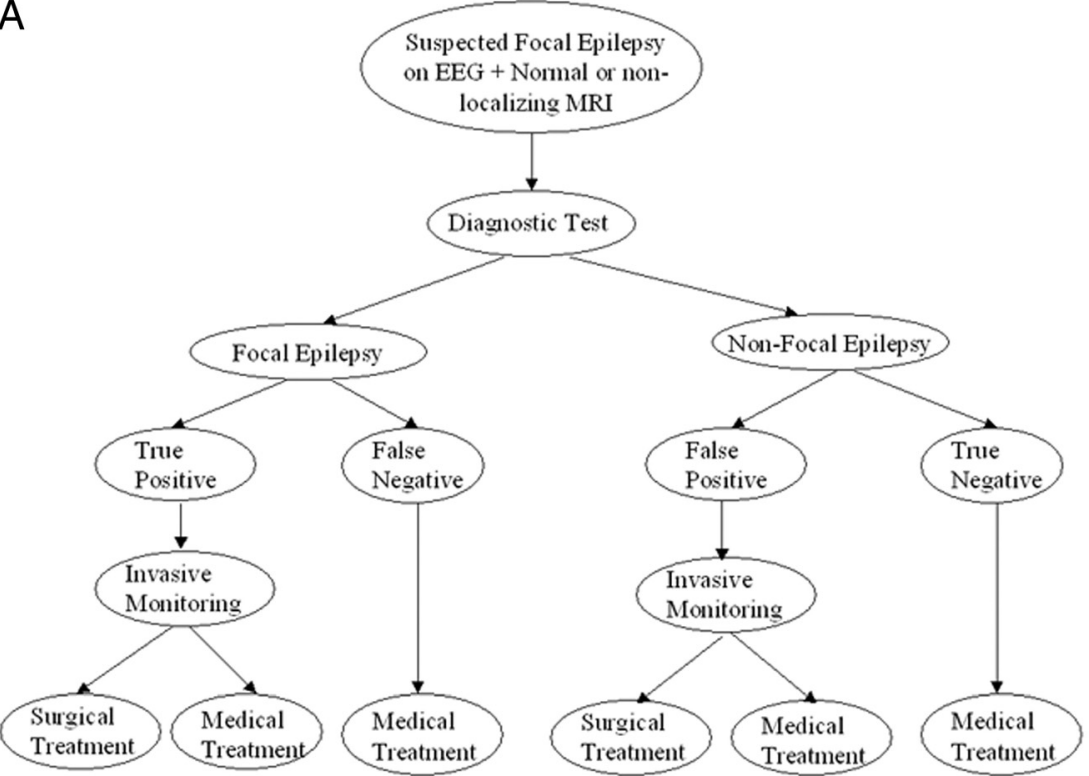

B

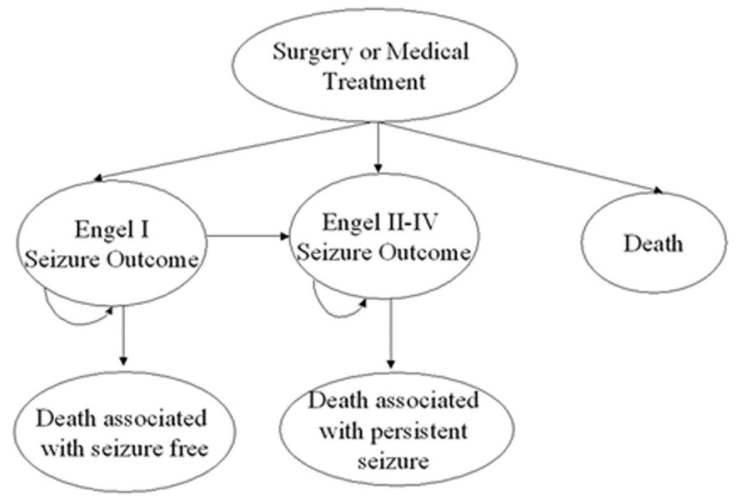

FIG 1. A, A schematic representation of the decision-analysis model in patients with suspected focal epilepsy on electroencephalography with normal MR imaging findings. B, The Markov model of the health states following surgical or medical treatment of patients with focal epilepsy.

will identify the epileptogenic zone in patients with suspected focal intractable epilepsy and normal MR imaging findings by using decision analysis.

\section{MATERIALS AND METHODS}

The Research Ethics Board of our institution does not require approval for studies based on literature review.

\section{Decision Model}

We used a Markov-based decision model to simulate the course of events of patients with suspected focal intractable epilepsy on video scalp EEG with normal MR imaging findings. We took the societal perspective to evaluate the cost-effectiveness of the diagnostic strategies. The base case was a 26-year-old, which was the mean age of the cohort undergoing these investigations for epilepsy surgery work-up in the study by Knowlton et al. ${ }^{16}$ The end point in the model was death or when the patient reached 100 years old. The lifetime of the base case was divided into 1-year cycles. Six diagnostic strategies consisting of 3 individual (PET, SPECT, and MEG) and 3 combinations of tests (PET+SPECT,
$\mathrm{PET}+\mathrm{MEG}$, and SPECT+MEG) were compared (Fig 1). If the test finding was positive, the patient would undergo invasive monitoring, followed by surgical resection or medical treatment. If the test finding was negative, the patient would continue with medical treatment.

Following surgery or medical treatment, the patient could enter 1 of the 3 health states: 1) Engel I seizure outcome or seizure-free, 2) Engel II-IV seizure outcome or persistent seizures, and 3) death (Fig 2). If the patient had Engel I outcome, the patient could continue in that health state or transition to Engel II-IV outcome or death. If the patient had Engel II-IV outcome, the patient could continue in that health state or transition to death.

The model was constructed and analyzed by using TreeAge Pro (TreeAge software, Williamstown, Massachusetts).

\section{Data and Model Assumptions}

Data sources for the model are shown in On-line Table 1. The probability of focal intractable epilepsy was derived from the literature. ${ }^{1-3}$

\section{Diagnostic Tests}

Sensitivity and specificity for the tests were obtained from Knowlton et al. ${ }^{16}$ We have chosen the results from this study for several reasons. First, this was a prospective study comparing the performance of these investigations with seizure-free surgical outcome. Second, all the tests individually and in combinations were conducted in the same cohort, and sensitivity and specificity for all these tests were reported. Third, this study included patients with both temporal and extratemporal lobe epilepsies.

We have not used sensitivity or specificity data from systematic reviews or meta-analysis because the reference standard included intracranial EEG or the specificity was not reported. Surgical outcome was considered a better reference standard than intracranial EEG because intracranial EEG coverage may not have included the epileptogenic zone. ${ }^{16}$ Systematic review for MEG reported a sensitivity of 0.84 (range, $0.20-1.0$ ) and a specificity of 0.52 (range, $0.06-1.0)^{17}$ compared with the reference standard of intracranial EEG and surgical outcome, with a huge range of sensitivity and specificity. Systematic review for PET reported that the sensitivity for PET was $84 \%$ (specificity $=86 \%$ ) for temporal lobe epilepsy and 33\% (specificity $=95 \%$ ) for extratemporal lobe epilepsy. ${ }^{18}$ More recently, another systematic review reported the positive predictive value of PET in temporal lobe epilepsy but did not report the negative predictive value and also did not include patients with extratemporal lobe epilepsy. ${ }^{19}$ Systematic review of 

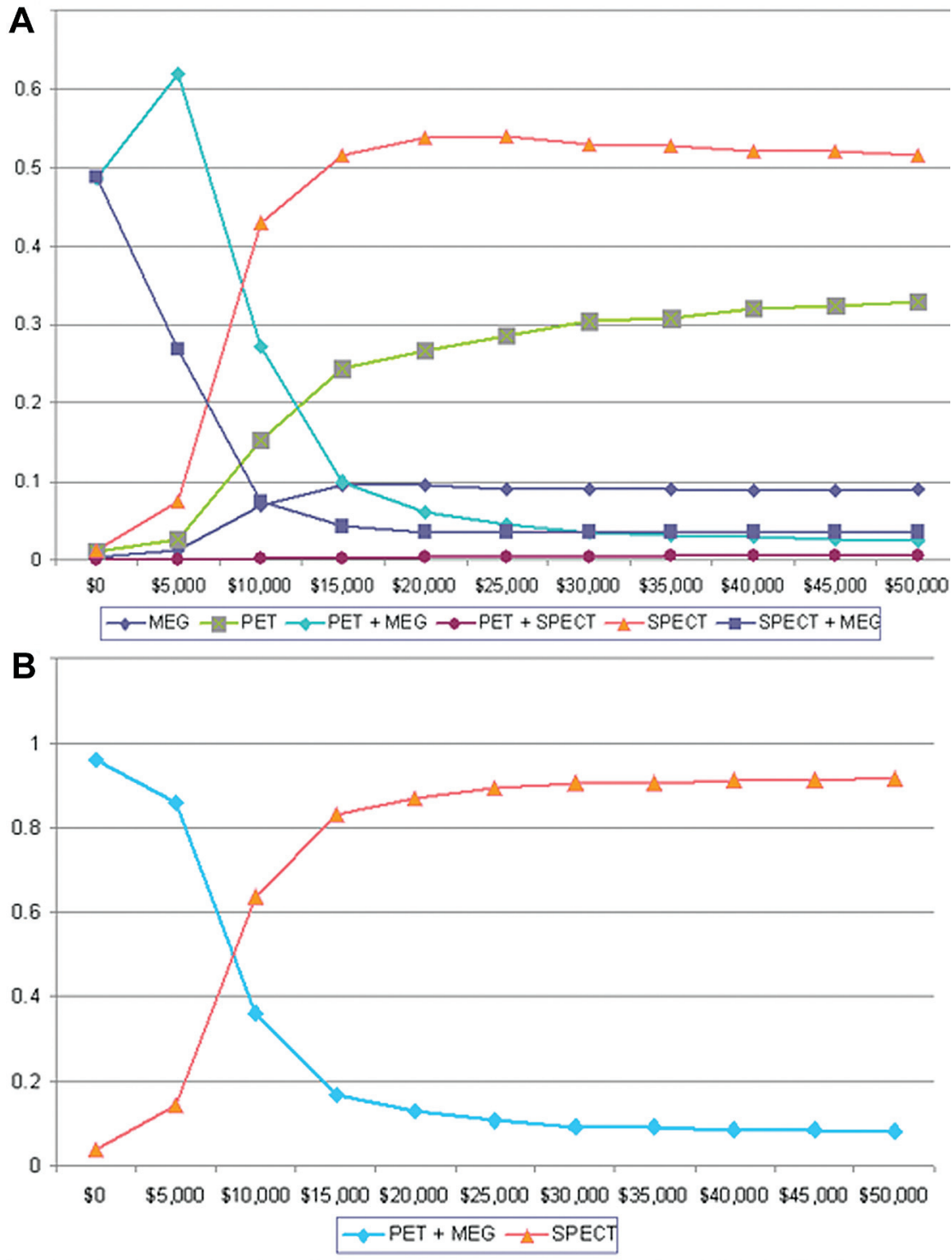

FIG 2. Cost-effectiveness acceptability curve of $6(A)$ strategies and PET+MEG and SPECT (B) strategies for a range of willingness to pay.

SPECT compared with EEG showed that in temporal lobe epilepsy, the sensitivity for ictal SPECT was $90 \%$ and the specificity was $73 \%$; in extratemporal lobe epilepsy, the sensitivity of ictal SPECT decreased to $81 \%$ and the specificity increased to $93 \% .{ }^{18}$ Subsequent meta-analysis on SPECT compared with surgical outcome ${ }^{20}$ reported a poorer sensitivity relative to an earlier systematic review that used intracranial EEG as the reference standard; the sensitivity of postictal SPECT was 0.787 (95\% CI, 0.643-0.893). However, the study did not report on the specificity of SPECT.

The probability of epilepsy surgery following invasive monitoring given that the patient has a true-positive test was $0.86 .{ }^{16}$ There were no data on the probability of epilepsy surgery following invasive monitoring for false-positive tests in the literature; the value was obtained by subtracting the probability of surgery following invasive monitoring, given a true-positive test $(0.86)$ by 1.0 .

\section{Utilities and Costs}

The utilities of the epilepsy health states were obtained from Choi et $a{ }^{21}$ who used standard gamble to derive preference-based quality-of-life values; standard gamble was then used to adjust life expectancy to obtain QALY following temporal lobe epilepsy surgery. The assumption was made that utilities were similar for extratemporal lobe epilepsy surgery. The utility of seizure-free was 0.96 and of persistent seizures was 0.75 .

The cost of the diagnostic tests was derived locally, because the costs of some of these investigations were not covered by provincial health insurance plans in Canada. The cost of PET and MEG was $\$ 1400$, and the cost of SPECT was $\$ 1500$. The cost of surgical and medical treatment was derived from direct and indirect costs estimated by Wiebe et al. ${ }^{22}$ Because these costs were based on the 1993 market price in Canadian dollars, the costs were revised to reflect the 2012 market price, by using the Canada-Consumer Price Index correction. ${ }^{23}$ The cost of surgical treatment was $\$ 43,537.09$ in the first year and $\$ 2066.64$ after the first year. The cost of medical treatment was $\$ 11,503.07$ in the first year and $\$ 4090.51$ after the first year.

\section{Surgical and Medical Seizure Outcomes}

The surgical outcomes were obtained from 2 meta-analysis studies reporting outcomes for 3 periods: $1-4$ years, 5-10 years, and $>10$ years following surgery for both temporal and extratemporal lobe epilepsies. ${ }^{10,11}$ There is no metaanalysis study on seizure outcomes following medical treatment for the same periods, to our knowledge. We used pooled results of seizure outcome by using the random effects model from 4 studies of $1-4$ years ${ }^{9,24-26}$ and from 2 studies of $5-10$ years ${ }^{27,28}$ following medical treatment. We did not identify any study reporting seizure outcome of $>10$ years following medical treatment. The rate of decline in seizure outcome in the surgical group between 5 and 10 years and at $>10$ years was assessed and applied to the medical group. The probability of seizure-free outcome following medical treatment that we used was similar to that reported in a meta-analysis for the follow-up period of $1-13$ years, which was $12 \% .^{29}$

\section{Mortality from Surgery and Excess Mortality from Epilepsy}

Death from epilepsy surgery is rare. ${ }^{10}$ Patients with epilepsy have a higher risk of premature death compared with the general population. ${ }^{7,8,30,31}$ For the general population age-specific mortality rates, we used the 2002 Canadian Life Tables. ${ }^{32}$ We used pooled estimates of the SMR for temporal lobe resections from Choi et $\mathrm{al}^{21}$ and assumed that the SMR was the same following temporal and extratemporal lobe resections. 


\begin{tabular}{|c|c|c|c|c|c|c|}
\hline \multirow[b]{2}{*}{ Strategies } & \multirow[b]{2}{*}{ Health Care Costs } & \multirow[b]{2}{*}{ Effectiveness (QALY) } & \multicolumn{2}{|c|}{ Difference } & \multirow[b]{2}{*}{$\operatorname{ICER}^{\mathrm{a}}(\$ / \mathrm{QALY})$} & \multirow[b]{2}{*}{ Dominance } \\
\hline & & & Costs & Effectiveness & & \\
\hline PET + MEG & $\$ 95,612$ & 16.30 & & - & - & \\
\hline SPECT + MEG & $\$ 95,824$ & 16.28 & $\$ 212^{b}$ & $-0.0241^{b}$ & - & Dominated \\
\hline PET + SPECT & $\$ 96,532$ & 16.36 & $\$ 920^{b}$ & $0.0543^{b}$ & $\$ 16,937^{c}$ & Ext. Dominated \\
\hline SPECT & $\$ 97,479$ & 16.45 & $\$ 1867^{b}$ & $0.1443^{b}$ & $\$ 12,934^{c}$ & \\
\hline MEG & $\$ 97,499$ & 16.42 & $\$ 21$ & $-0.0238^{d}$ & - & Dominated \\
\hline PET & $\$ 97,623$ & 16.44 & $\$ 144$ & $-0.0121^{d}$ & - & Dominated \\
\hline
\end{tabular}

Note:- - indicates XXX; Ext., extended.

a The results of incremental effectiveness and ICER may not show as the calculated value based on the "Effectiveness" column due to round-up.

b The result of incremental cost and incremental effectiveness was compared with strategy 1-PET + MEG.

${ }^{c}$ The ICER was calculated when the strategies were compared with strategy 1-PET+MEG.

${ }^{d}$ The results of incremental effectiveness were compared with strategy 4 -SPECT.

\section{Discounting}

Both costs and utilities were discounted at an annual rate of $3 \%$.

\section{Sensitivity Analyses}

To assess the impact of statistical uncertainty around key model inputs, we performed 1-way and probabilistic sensitivity analysis.

One-Way Sensitivity Analysis. We varied the sensitivity and specificity of single and combined test strategies, seizure-free outcomes following surgery and medical treatment, the SMR for seizure-free and persistent seizure states, the cost of tests and treatment, the utility of each health state, the probability of focal epilepsy, the probability of surgery after invasive monitoring, and the discount rate. The range of assumptions tested is shown in On-line Table 1.

Probabilistic Sensitivity Analysis. Probabilistic sensitivity analysis was performed by using Monte Carlo simulations with 10,000 iterations, and all the parameters were varied simultaneously by using $\beta$, log-normal, and $\gamma$ distributions (On-line Table 1). If the resulting ICER of the more effective strategy was less than the societal willingness to pay, then that iteration was considered cost-effective. Probabilistic sensitivity analysis results were presented as a cost-effectiveness acceptability curve with a series of societal willingness to pay.

\section{RESULTS}

\section{Base Case}

The health care cost of the baseline strategy (PET+MEG, with corresponding treatment) was estimated at \$95,612 with an expected 16.30 QALY gained (Table 1). The health care cost of the SPECT strategy was $\$ 97,479$ with 16.45 QALY gained, resulting in an ICER of $\$ 12,934$ per QALY compared with PET+MEG. A strategy that is more effective and less costly than competing strategies is the dominant strategy. On the basis of the domination algorithm, SPECT + MEG was dominated by PET+MEG because PET + MEG could provide better QALY with lower costs. Similarly, the MEG-alone and PET-alone strategies were dominated by the SPECT-alone strategy. After eliminating the dominated strategies, the model identified PET+MEG, PET+SPECT, and SPECT as the cost-effective strategies, with PET +MEG as the baseline strategy. The ICER of SPECT was lower than the ICER of PET+SPECT; therefore, PET + SPECT was extended dominated by SPECT and was eliminated from the cost-effective strategies. In the base case, PET+MEG and SPECT were the 2 preferred strategies. When these 2 strategies were compared, PET + MEG was preferred relative to SPECT.

\section{Sensitivity Analyses}

One-Way Sensitivity Analyses. Results of 1-way sensitivity analyses are shown in On-line Table 2. The decision of the model was sensitive to variations in the sensitivity and specificity of each testing strategy. When the sensitivity or specificity of PET was increased to the upper bound of the $95 \%$ confidence interval, PET became an additional dominant strategy. When the sensitivity or specificity of SPECT + MEG was reduced to the lower bound of the $95 \%$ confidence interval, the decision of the model was also altered and SPECT + MEG became an additional dominant strategy to PET+MEG and SPECT.

Two other variables have been shown to affect the decision of the model. When the probability of focal epilepsy increased, SPECT + MEG and SPECT became the 2 preferred strategies. Similarly, when the probability of surgery following invasive monitoring given that the patient has focal epilepsy increased, SPECT + MEG and SPECT became the preferred strategies.

For the range of assumptions tested on the costs of surgical and medical treatment, the SMR for seizure-free and persistent seizures, the utility of health states, seizure-free outcomes following surgery and medical treatment, and the discount rate, PET+MEG and SPECT remained the preferred strategies. Because these variables were common elements in all strategies, the variation of the variables did not alter the direction of the decision but changed the value of the ICER, which reflects the magnitude of the variation of those variables. The variation of these parameters caused the ICER of SPECT compared with PET+MEG baseline strategy to change from $\$ 5919$ to $\$ 59,107$. Varying the costs of each diagnostic test strategy also did not alter the decision, and PET + MEG and SPECT remained the preferred strategies.

Probabilistic Sensitivity Analyses. Figure $2 A$ shows the cost-effectiveness acceptability curve for all 6 strategies for a range of willingness to pay. When the willingness to pay was low, PET+MEG was the preferred strategy. However, as the willingness to pay increased, SPECT became the preferred strategy. The 2 cost-effectiveness acceptability curves crossed at $\$ 10,000$, indicating that if the decisionmaker was willing to pay more than $\$ 10,000$ per QALY gained, SPECT was more cost-effective than PET+MEG.

The cost-effectiveness acceptability curve of SPECT was close 
to $90 \%$ when the willingness to pay increased to $\$ 30,000$ per QALY gained (Fig 2B). Due to potential for false-positive results, there was an approximately $10 \%$ chance that SPECT would never be considered cost-effective even when the willingness to pay increased to infinity.

\section{DISCUSSION}

This is the first study that assessed the cost-effectiveness of 3 widely used clinical diagnostic tests to identify the epileptogenic zone. We have found in the base case that PET + MEG and SPECT were the cost-effective strategies to identify the epileptogenic zone in patients with suspected focal intractable epilepsy with normal MR imaging findings. SPECT had the lowest ICER of \$12,934 per QALY gained compared with PET+MEG, the baseline strategy. The decision of the model remained unchanged for a wide range of uncertainties tested, with the exception of the varying sensitivity or specificity of the tests.

One study evaluated the cost-savings of FDG-PET relative to ictal SPECT and combined video EEG + MR imaging for Engel class I/II outcomes. ${ }^{33}$ Patients with concordant video EEG and MR imaging abnormalities would undergo surgical treatment, but those with discordant findings would undergo medical therapy. The authors found that EEG + MR imaging had the lowest cost per class I/II outcomes compared with PET and SPECT, individually. We have not included video EEG + MR imaging as one of the strategies because there are no sensitivity or specificity data on video EEG and normal MR imaging findings in the literature. Furthermore, patients with normal or nonlocalizing MR imaging findings and suspected focal epilepsy would usually undergo further tests before surgery in clinical practice. The sensitivity and specificity of the tests used in our model were derived from a study that evaluated this particular group of patients with normal MR imaging findings. ${ }^{16}$ We have included combined test strategies within the model because they are performed in clinical practice in patients with normal MR imaging findings. We have also evaluated the cost-effectiveness of diagnostic tests by using the ICER rather than cost savings.

O'Brien et $\mathrm{al}^{33}$ found that PET consistently detected the highest proportion of class I/II outcomes unless the sensitivity of ictal SPECT exceeded that of PET. In their study, the sensitivity of PET was 0.96 (range, 0.805-0.993) and the specificity was 0.246 (range, $0.158-0.363)^{33}$; the sensitivity was higher than that reported in the literature for a combination of temporal and extratemporal lobe epilepsies. ${ }^{18}$ The sensitivity and specificity of PET that we used ${ }^{16}$ were intermediate for temporal and extratemporal lobe epilepsies. ${ }^{18}$ In our model, the sensitivity and specificity of PET were slightly lower than those for SPECT. In the study by O'Brien et $\mathrm{al},{ }^{33}$ the differences in sensitivity and specificity of PET and SPECT were larger compared with those in our study. These differences could have contributed to differences in the base case findings between the 2 studies. We have found that the decision of the model was sensitive to variations in the sensitivity and specificity of the tests. We used findings from Knowlton et $\mathrm{al}^{16}$ for reasons detailed in the "Materials and Methods" section. However, the sample size of the study was relatively small, resulting in a relatively large confidence interval of the sensitivity and speci- ficity of the tests. ${ }^{16}$ This highlights the need for a further study with a larger sample size to improve the precision of the estimates.

An intervention or test is considered cost-effective if the ICER falls below the societal willingness-to-pay threshold, which is usually set at $\$ 50,000$ (in US dollars) per QALY. ${ }^{34}$ The ICER of SPECT (\$12,934 per QALY gained) relative to PET+MEG was lower compared with that of diagnostic tests for other conditions. The ICER of sonography+CT was $\$ 17,108$ per QALY relative to sonography, while the ICER of CT alone was $\$ 26,260$ per QALY relative to sonography for acute appendicitis in children. ${ }^{35}$ The ICER of CTA with 70\%-99\% cutoff was $€ 71,419$ per QALY compared with a baseline strategy of duplex sonography+CTA for investigating transient ischemic attacks. ${ }^{36}$ Biennial screening with mammography for breast cancer from 40-69 years of age had an ICER of \$61,600 per QALY saved compared with no screening. ${ }^{37}$

Our study had several limitations. There were limited seizure outcome data in the medically treated patients in the literature, particularly long-term outcome data. The assumption was made that the decline in long-term seizure outcome was the same in both surgical and medical groups. Even if one allowed uncertainties in the estimate of long-term seizure outcome in the medical group, the preferred strategies (PET+MEG and SPECT) remained unchanged. We have not included the morbidity of surgical treatment in the model because complications such as infection and hemorrhage are usually transient ${ }^{9,10}$ and short-term morbidity is unlikely to have a major impact on long-term QALY. We have also not included the 3 combined tests within the model for several reasons. The sensitivity $(8 \%)$ of PET + SPECT + MEG was the lowest, and the specificity (100\%) was the highest among all the tests combined. When the test strategy has $100 \%$ specificity and the disorder is relatively uncommon, the strategy will always dominate. When the sensitivity of the test is very low, such as in $\mathrm{PET}+\mathrm{SPECT}+\mathrm{MEG}$, most patients would be excluded from surgical treatment. In clinical practice, some patients who have 2 of 3 tests that were localizing may still undergo invasive monitoring and epilepsy surgery. However, there are insufficient data to estimate the likelihood of this occurring. We did not include advanced MR imaging techniques such as MR spectroscopy, perfusion, or diffusion tensor imaging as a potential strategy because these techniques are not routinely used in clinical practice to identify the epileptogenic zone. Furthermore, the sensitivity and specificity of these MR imaging techniques for identifying the epileptogenic zone are not available in the literature.

\section{CONCLUSIONS}

The decision on which diagnostic test strategies to choose depends on many factors, including local expertise and local availability of diagnostic tests. PET and SPECT are widely available in most epilepsy centers in North America and Europe. MEG is not as widely available; however, it is increasingly used in epilepsy centers. If all 3 tests were available, our study suggested that the choice was dependent on the sensitivity and specificity of the tests. In the base case, PET+MEG and SPECT were the preferred strategies. The choice between these 2 strategies was dependent on the willingness to pay. When the willingness to pay was low, PET+MEG was the favored strategy; as the willingness to pay increased, SPECT became the preferred strategy. 
Disclosures: Elysa Widjaja—RELATED: Grant: GE-Association of University Radiologists (AUR), ${ }^{*}$ UNRELATED: Grants/Grants Pending: Sickkids Foundation/Institute for Human Development, Child and Youth Health-Canadian Institutes of Health Research.* L. Santiago Medina—UNRELATED: Royalties: books from Springer on evidence-based imaging *Money paid to the institution.

\section{REFERENCES}

1. Berg AT, Shinnar S, Levy SR, et al. Early development of intractable epilepsy in children: a prospective study. Neurology 2001;56:1445-52

2. Kwan P, Brodie MJ. Early identification of refractory epilepsy. N Engl J Med 2000;342:314-19

3. Engel J Jr. Etiology as a risk factor for medically refractory epilepsy: a case for early surgical intervention. Neurology 1998;51:1243-44

4. Leidy NK, Elixhauser A, Vickrey B, et al. Seizure frequency and the health-related quality of life of adults with epilepsy. Neurology 1999;53:162-66

5. Taylor RS, Sander JW, Taylor RJ, et al. Predictors of health-related quality of life and costs in adults with epilepsy: a systematic review. Epilepsia 2011;52:2168-80

6. Neligan A, Bell GS, Johnson AL, et al. The long-term risk of premature mortality in people with epilepsy. Brain 2011;134:388-95

7. Nilsson L, Ahlbom A, Farahmand BY, et al. Mortality in a populationbased cohort of epilepsy surgery patients. Epilepsia 2003;44:575-81

8. Nashef L, Fish DR, Sander JW, et al. Incidence of sudden unexpected death in an adult outpatient cohort with epilepsy at a tertiary referral centre. J Neurol Neurosurg Psychiatry 1995;58:462-64

9. Wiebe S, Blume WT, Girvin JP, et al. A randomized, controlled trial of surgery for temporal-lobe epilepsy. N Engl J Med 2001;345:311-18

10. Engel J Jr, Wiebe S, French J, et al. Practice parameter: temporal lobe and localized neocortical resections for epilepsy: report of the Quality Standards Subcommittee of the American Academy of Neurology, in association with the American Epilepsy Society and the American Association of Neurological Surgeons. Neurology 2003;60:538-47

11. Téllez-Zenteno JF, Dhar R, Wiebe S. Long-term seizure outcomes following epilepsy surgery: a systematic review and meta-analysis. Brain 2005;128:1188-98

12. Engel J Jr, McDermott MP, Wiebe S, et al. Early surgical therapy for drug-resistant temporal lobe epilepsy: a randomized trial. JAMA 2012;307:922-30

13. Téllez-Zenteno JF, Hernandez Ronquillo L, Moien-Afshari F, et al. Surgical outcomes in lesional and non-lesional epilepsy: a systematic review and meta-analysis. Epilepsy Res 2010;89:310-18

14. Immonen A, Jutila L, Muraja-Murro A, et al. Long-term epilepsy surgery outcomes in patients with MRI-negative temporal lobe epilepsy. Epilepsia 2010;51:2260-69

15. Bien CG, Szinay M, Wagner J, et al. Characteristics and surgical outcomes of patients with refractory magnetic resonance imagingnegative epilepsies. Arch Neurol 2009;66:1491-99

16. Knowlton RC, Elgavish RA, Bartolucci A, et al. Functional imaging: II. Prediction of epilepsy surgery outcome. Ann Neurol 2008;64:35-41

17. Lau M, Yam D, Burneo JG. A systematic review on MEG and its use in the presurgical evaluation of localization-related epilepsy. Epilepsy Res 2008;79:97-104
18. Spencer SS. The relative contributions of MRI, SPECT, and PET imaging in epilepsy. Epilepsia 1994;35(suppl 6):S72-89

19. Willmann O, Wennberg R, May T, et al. The contribution of $\mathbf{1 8 F}$ FDG PET in preoperative epilepsy surgery evaluation for patients with temporal lobe epilepsy" a meta-analysis. Seizure 2007;16: 509-20

20. Devous MD Sr, Thisted RA, Morgan GF, et al. SPECT brain imaging in epilepsy: a meta-analysis. J Nucl Med 1998;39:285-93

21. Choi H, Sell RL, Lenert L, et al. Epilepsy surgery for pharmacoresistant temporal lobe epilepsy: a decision analysis. JAMA 2008; 300:2497-505

22. Wiebe S, Blume WT, Girven JP, et al. An economic evaluation of surgery for temporal lobe epilepsy. J Epilepsy 1995;8:227-35

23. Canada-Consumer Price Index (CPI) History: 2001-2012. http:// www.rateinflation.com/consumer-price-index/canada-historicalcpi.php?form = cancpi. Accessed December 2011

24. Chen LS, Wang N, Lin MI. Seizure outcome of intractable partial epilepsy in children. Pediatr Neurol 2002;26:282-87

25. Widjaja E, Li B, Schinkel CD, et al. Cost-effectiveness of pediatric epilepsy surgery compared to medical treatment in children with intractable epilepsy. Epilepsy Res 2011;94:61-68

26. Selwa LM, Schmidt SL, Malow BA, et al. Long-term outcome of nonsurgical candidates with medically refractory localization-related epilepsy. Epilepsia 2003;44:1568-72

27. Carreño M, Becerra JL, Castillo J, et al. Seizure frequency and social outcome in drug resistant epilepsy patients who do not undergo epilepsy surgery. Seizure 2011;20:580-82

28. Bien CG, Schulze-Bonhage A, Soeder BM, et al. Assessment of the long-term effects of epilepsy surgery with three different reference groups. Epilepsia 2006;47:1865-69

29. Schmidt D, Stavem K. Long-term seizure outcome of surgery versus no surgery for drug-resistant partial epilepsy: a review of controlled studies. Epilepsia 2009;50:1301-09

30. Annegers JF, Coan SP, Hauser WA, et al. Epilepsy, vagal nerve stimulation by the NCP system, mortality, and sudden, unexpected, unexplained death. Epilepsia 1998;39:206-12

31. Sperling MR, Feldman H, Kinman J, et al. Seizure control and mortality in epilepsy. Ann Neurol 1999;46:45-50

32. Canadian Life Tables: 2002. www.statcan.gc.ca/pub/84-537-x/4064441eng.htm. Accessed December 2011

33. O'Brien TJ, Miles K, Ware R, et al. The cost-effective use of 18F-FDG PET in the presurgical evaluation of medically refractory focal epilepsy. J Nucl Med 2008;49:931-37

34. King JT Jr, Tsevat J, Lave JR, et al. Willingness to pay for a quality adjusted life year: implications for societal health care resource allocation. Med Decis Making 2005;25:667-77

35. Wan MJ, Krahn M, Ungar WJ, et al. Acute appendicitis in young children: cost-effectiveness of US versus CT in diagnosis-a Markov decision analytic model. Radiology 2009;250:378-86

36. Tholen AT, de Monye C, Genders TS, et al. Suspected carotid artery stenosis: cost-effectiveness of CT angiography in work-up of patients with recent TIA or minor ischemic stroke. Radiology 2010;256:585-97

37. Wong IO, Kuntz KM, Cowling BJ, et al. Cost effectiveness of mammography screening for Chinese women. Cancer 2007;110:885-95 